MATHEMATICS OF COMPUTATION

Volume 72 , Number 242 , Pages 697-713

S 0025-5718(02)01472-2

Article electronically published on October 29, 2002

\title{
ABOUT THE SHARPNESS OF THE STABILITY ESTIMATES IN THE KREISS MATRIX THEOREM
}

\author{
M. N. SPIJKER, S. TRACOGNA, AND B. D. WELFERT
}

\begin{abstract}
One of the conditions in the Kreiss matrix theorem involves the resolvent of the matrices $A$ under consideration. This so-called resolvent condition is known to imply, for all $n \geq 1$, the upper bounds $\left\|A^{n}\right\| \leq e K(N+1)$ and $\left\|A^{n}\right\| \leq e K(n+1)$. Here $\|\cdot\|$ is the spectral norm, $K$ is the constant occurring in the resolvent condition, and the order of $A$ is equal to $N+1 \geq 1$.

It is a long-standing problem whether these upper bounds can be sharpened, for all fixed $K>1$, to bounds in which the right-hand members grow much slower than linearly with $N+1$ and with $n+1$, respectively. In this paper it is shown that such a sharpening is impossible. The following result is proved: for each $\epsilon>0$, there are fixed values $C>0, K>1$ and a sequence of $(N+1) \times(N+1)$ matrices $A_{N}$, satisfying the resolvent condition, such that $\left\|\left(A_{N}\right)^{n}\right\| \geq C(N+1)^{1-\epsilon}=C(n+1)^{1-\epsilon}$ for $N=n=1,2,3, \ldots$.

The result proved in this paper is also relevant to matrices $A$ whose $\epsilon$-pseudospectra lie at a distance not exceeding $K \epsilon$ from the unit disk for all $\epsilon>0$.
\end{abstract}

\section{INTRODUCTION}

1.1. The Kreiss matrix theorem. In the following we use the notation

$$
|\mathbf{x}|=\left(\left|x_{0}\right|^{2}+\left|x_{1}\right|^{2}+\cdots+\left|x_{N}\right|^{2}\right)^{1 / 2}
$$

for vectors $\mathbf{x} \in \mathbb{C}^{N+1}$ with components $x_{0}, x_{1}, \ldots, x_{N}$. Further, we define

$$
\|A\|=\max \left\{|A \mathbf{x}|: \mathbf{x} \in \mathbb{C}^{N+1} \text { with }|\mathbf{x}|=1\right\}
$$

for square matrices $A$ of order $N+1$.

In the stability analysis of numerical processes, one is often faced with the problem of estimating $\left\|A^{n}\right\|$ for $n \geq 1$; stable numerical processes are distinguished by the property that $\left\|A^{n}\right\|$ is of moderate size.

Kreiss (1962) established an important theorem, called the Kreiss matrix theorem, which has been one of the fundamental results for establishing numerical stability. Still recently, much research was devoted to this theorem and variants thereof (see, e.g., Giles (1997), Kraaijevanger (1994), Lubich \& Nevanlinna (1991), Reddy \& Trefethen (1992), Spijker \& Straetemans (1996, 1997), Strikwerda \& Wade (1991), Toh \& Trefethen (1999), and the review papers Borovykh \& Spijker (2000), Dorsselaer et al. (1993), Nevanlinna (1997), Strikwerda \& Wade (1997)).

Received by the editor May 12, 1998.

2000 Mathematics Subject Classification. Primary 15A60, 65M12.

Key words and phrases. Kreiss matrix theorem, resolvent condition, stability estimate, numerical stability, $\epsilon$-pseudospectrum. 
One of the (equivalent) conditions occurring in the Kreiss theorem involves the so-called resolvent $(z I-A)^{-1}$ of $A$; here $z$ is a complex variable and $I$ denotes the identity matrix of order $N+1$. This condition requires that a constant $K \geq 1$ exists such that

$$
\left\{\begin{array}{l}
z I-A \text { is invertible } \\
\left\|(z I-A)^{-1}\right\| \leq \frac{K}{|z|-1} \text { for all } z \in \mathbb{C} \text { with }|z|>1 .
\end{array}\right.
$$

We shall refer to (1.1) as a Kreiss resolvent condition, and to $K$ as a Kreiss constant, for the matrix $A$.

The current form of the Kreiss theorem implies that both estimates

$$
\left\|A^{n}\right\| \leq e K(N+1) \quad \text { for } n, N \geq 0
$$

and

$$
\left\|A^{n}\right\| \leq e K(n+1) \quad \text { for } n, N \geq 0
$$

are valid whenever $A$ is a matrix of order $N+1$ satisfying (1.1) (see Spijker (1991) and Wegert \& Trefethen (1994)). The purpose of this paper is to contribute to the important question of how far these estimates are sharp when $K$ is an arbitrary fixed number.

For the sake of completeness we mention that (1.1) can be reformulated in terms of the so-called $\epsilon$-pseudospectrum $\Lambda_{\epsilon}(A)=\{\lambda: \lambda$ is an eigenvalue of some $(N+1) \times(N+1)$ matrix $B$ with $\|B-A\| \leq \epsilon\}$ : condition (1.1) is equivalent to the requirement that, for all $\epsilon>0$, the set $\Lambda_{\epsilon}(A)$ lies within the disk $\{z:|z| \leq 1+K \epsilon\}$ (see Reddy \& Trefethen $(1990,1992))$.

1.2. Known results about the sharpness of (1.2)-(1.3). LeVeque \& Trefethen (1984) constructed $(N+1) \times(N+1)$ matrices $A_{N}$, satisfying (1.1) with $K=K_{N}$ (for $N \geq 0$ ), such that

$$
\lim _{N \rightarrow \infty} \frac{\left\|\left(A_{N}\right)^{N}\right\|}{(N+1) K_{N}}=e .
$$

Here, the Kreiss constants $K_{N}$ are such that $K_{N} \rightarrow \infty$ (when $N \rightarrow \infty$ ). This counterexample proves that the constant $e$, occurring in the right-hand members of the estimates (1.2)-(1.3) cannot be replaced by any smaller constant-provided the estimates are required to follow from (1.1) for all $K \geq 1$.

Clearly, this interesting counterexample is not relevant to the question mentioned in Section 1.1 about the sharpness of (1.2)-1.3 for arbitrary fixed $K$. In fact, for $K=1$, the Kreiss resolvent condition is known to imply

$$
\left\|A^{n}\right\| \leq 2 \quad \text { for } n, N \geq 0
$$

(see, e.g., Dorsselaer et al. (1993)), which is a much stronger estimate than (1.2)(1.3) with $K=1$.

It is a long-standing problem in how far, also for all fixed $K$ greater than 1 , the estimates (1.2)-(1.3) can be sharpened, say to

$$
\left\|A^{n}\right\| \leq a(N, K) \quad \text { for } n, N \geq 0
$$

and

$$
\left\|A^{n}\right\| \leq b(n, K) \quad \text { for } n, N \geq 0,
$$


where $a(N, K)$ and $b(n, K)$ grow (much) slower than linearly with $N$ and $n$, respectively. An early contribution was made by McCarthy \& Schwartz (1965), who proved that $a(N, K)$ must grow faster than $(\log N)^{\beta}$ for any $\beta<1 / 4$, when $K$ is fixed with $K>\pi+1$. Later, Shields (1978) addressed a related issue leading to the question of whether $b(n, K)$, for each $K>1$, can be chosen to be equal to $c(K) \sqrt{n+1}$, with $c(K)$ only depending on $K$. He presented some evidence for the answer to this question to be positive.

The most recent contribution toward the solution of the above problem, known to the authors, was made by J. van Dorsselaer (see Dorsselaer et al. (1993, Theorem 2.8)). His contribution implies the following theorem.

Theorem 1.1. Suppose (1.1) implies (1.4)-(1.5). Let $K>\pi+1$ be given. Then there is a positive constant $C$ such that

$$
a(N, K) \geq C \frac{\sqrt{\log N}}{\log (\log N)}, \quad b(n, K) \geq C \frac{\sqrt{\log n}}{\log (\log n)} \quad \text { for } n, N \geq 2 .
$$

For discussions of various sharpness questions which have (some) similarity to the one above, we refer to Dorsselaer et al. (1993), Kraaijevanger (1994), Lubich \& Nevanlinna (1991), Sand (1996), Spijker \& Straetemans (1996), Strikwerda \& Wade (1991), Toh \& Trefethen (1999).

1.3. Formulation of the main result of the paper. There is a considerable gap between the growth (which is linear with $N+1$ and with $n+1$ ) of the upper bounds in (1.2)-(1.3) and the very slow growth of the lower bounds for $a(N, K)$ and $b(n, K)$ in Theorem 1.1 Corollary 1.3 below almost bridges this gap by giving, for any $\epsilon>0$, lower bounds for $a(N, K)$ and $b(n, K)$ which grow like $(N+1)^{1-\epsilon}$ and $(n+1)^{1-\epsilon}$, respectively. Moreover, this corollary completely settles the abovementioned question originating from Shields (1978): the answer to this question is negative.

The main theorem of our paper is as follows.

Theorem 1.2. Let $\epsilon>0$ be given. Then constants $K>1$ and $C>0$ exist such that, for each $N \geq 0$, there is an $(N+1) \times(N+1)$ matrix A satisfying both (1.1) and the inequality

$$
\left\|A^{N}\right\| \geq C(N+1)^{1-\epsilon} .
$$

Clearly, Theorem 1.2 implies the following corollary.

Corollary 1.3. Suppose (1.1) implies (1.4)-(1.5). Let $\epsilon>0$ be given. Then there exist fixed values $K>1$ and $C>0$ such that

$$
a(N, K) \geq C(N+1)^{1-\epsilon}, \quad b(n, K) \geq C(n+1)^{1-\epsilon} \quad \text { for } n, N \geq 0 .
$$

1.4. Outline of rest of the paper. The purpose of the following sections is to prove Theorem 1.2 .

In Section 2 we deal with an $(N+1) \times(N+1)$ matrix $A$ which is still of a rather general form. We formulate conditions on the eigenvectors of this matrix which imply both (1.1) and a lower bound for $\left\|A^{N}\right\|$. The main ideas in this section were used earlier by McCarthy \& Schwartz (1965), Kraaijevanger (1994) and Spijker \& Straetemans (1996).

In Section 3 we focus on the case where the matrix A, considered in Section 2, has a set of eigenvectors constituting a lower triangular Toeplitz matrix $T=\left(d_{i-j}\right)$ of 
order $N+1$. We relate arbitrary vectors $\mathbf{c} \in \mathbb{C}^{N+1}$, with components $c_{0}, c_{1}, \ldots, c_{N}$, to square integrable functions $f$ on $[-\pi, \pi]$, with Fourier coefficients $\widehat{f}(\ell)=c_{\ell}$ (for $0 \leq \ell \leq N)$. In doing so, we can reduce the conditions of Section 2 to conditions involving only a function $g$ with $\widehat{g}(\ell)=d_{\ell}(0 \leq \ell \leq N)$.

In Section 4 we specify the $(N+1) \times(N+1)$ matrix $A$ completely. We choose the entries $d_{i-j}$ of $T$ such that the corresponding function $g$ satisfies the conditions of Section 3 in a convenient manner. With this choice for $A$ we can conclude both that (1.1) holds and that a lower bound of the desired form holds for $\left\|A^{N}\right\|$.

\section{Conditions on a general matrix $A$}

In this section we consider a matrix $A$ of order $N+1$ of the form

$$
A=T \Lambda T^{-1}
$$

where $\Lambda$ is the diagonal matrix

$$
\Lambda=\left[\begin{array}{ccc}
\lambda_{0} & & \\
& \ddots & \\
& & \lambda_{N}
\end{array}\right] \text { with } \lambda_{\ell}=e^{\frac{i \ell \pi}{N}} \text { for } \ell=0, \ldots, N
$$

and $T$ is a nonsingular matrix (in the case $N=0$ set $\lambda_{0}=1$ ). The form (2.1) of $A$ allows for an easy determination of an expression for the powers of $A$, while the particular choice (2.2) for the matrix $\Lambda$ enables us to apply a nice result by McCarthy \& Schwartz (1965) and reduce the Kreiss resolvent condition (1.1) to a set of more tractable conditions on the columns of $T$.

In order to express this result, we write the matrices $T$ and $T^{-1}$ in the form

$$
T=\left[\begin{array}{l|l|l} 
& & \\
\mathbf{u}_{0} & \cdots & \mathbf{u}_{N}
\end{array}\right] \text { and } T^{-1}=\left[\begin{array}{c}
\frac{\mathbf{v}_{0}^{T}}{\vdots} \\
\hline \mathbf{v}_{N}^{T}
\end{array}\right]
$$

respectively. Note that $\mathbf{u}_{0}, \ldots, \mathbf{u}_{N}$ are eigenvectors of $A$. Let

$$
P_{j}=\sum_{\ell=0}^{j} \mathbf{u}_{\ell} \mathbf{v}_{\ell}^{T} \quad \text { for } j=0, \ldots, N .
$$

In particular $P_{N}=T T^{-1}=I$. More generally, the identity $T^{-1} T=I$ yields

$$
\mathbf{v}_{k}^{T} \mathbf{u}_{\ell}= \begin{cases}1 & \text { if } k=\ell \\ 0 & \text { otherwise }\end{cases}
$$

so that $P_{j}^{2}=P_{j}$ for $j=0, \ldots, N$, i.e., $P_{j}$ is a projection matrix onto the subspace of $\mathbb{C}^{N+1}$ spanned by the columns $\mathbf{u}_{0}, \ldots, \mathbf{u}_{j}$ of $T$.

Lemma 2.1 (McCarthy \& Schwartz, 1965). Assume that there exists a constant $\beta$ such that

$$
\left\|P_{j}\right\| \leq \beta \quad \text { for every } j=0, \ldots, N \text {. }
$$

Then the matrix A given by (2.1) -(2.2) satisfies the Kreiss resolvent condition (1.1) with $K=\beta \pi+1$. 
Proof. Using the notations of (2.3)-(2.4) we write $\mathbf{u}_{j} \mathbf{v}_{j}^{T}=P_{j}-P_{j-1}$ for $j=$ $1, \ldots, N$. Hence

$$
\begin{aligned}
(z I-A)^{-1} & =T(z I-\Lambda)^{-1} T^{-1} \\
& =\sum_{j=0}^{N} \frac{1}{z-\lambda_{j}} \mathbf{u}_{j} \mathbf{v}_{j}^{T} \\
& =\sum_{j=0}^{N-1}\left(\frac{1}{z-\lambda_{j}}-\frac{1}{z-\lambda_{j+1}}\right) P_{j}+\frac{1}{z-\lambda_{N}} I \\
& =\sum_{j=0}^{N-1}\left(\phi\left(\lambda_{j}\right)-\phi\left(\lambda_{j+1}\right)\right) P_{j}+\frac{1}{z-\lambda_{N}} I
\end{aligned}
$$

where $\phi(\lambda)=(z-\lambda)^{-1}$. For fixed $z$ such that $|z|>1$ and $|\lambda|=1$ we have

$$
\left|\phi(\lambda)-\frac{\bar{z}}{|z|^{2}-1}\right|=\left|\frac{\bar{z} \lambda-1}{(z-\lambda)\left(|z|^{2}-1\right)}\right|=\left|\frac{\bar{z}-\bar{\lambda}}{(z-\lambda) \bar{\lambda}}\right| \frac{1}{|z|^{2}-1}=\frac{1}{|z|^{2}-1},
$$

i.e., $\phi$ maps the unit circle to the circle $\mathcal{C}_{z}$ of center $\frac{\bar{z}}{|z|^{2}-1}$ and radius $\frac{1}{|z|^{2}-1}$ $(\bar{z}$ denotes the complex conjugate of $z$ ). Moreover $\phi$ has winding number 1 . Hence $\sum_{j=0}^{N-1}\left|\phi\left(\lambda_{j}\right)-\phi\left(\lambda_{j+1}\right)\right|$ is the length of a polygonal curve inscribed in $\mathcal{C}_{z}$, which is less than the circumference $\frac{2 \pi}{|z|^{2}-1}$ of $\mathcal{C}_{z}$. Finally we obtain, using (2.6),

$$
\left\|(z I-A)^{-1}\right\| \leq \frac{2 \beta \pi}{|z|^{2}-1}+\frac{1}{|z|-1}=\frac{1}{|z|-1}\left(\frac{2 \beta \pi}{|z|+1}+1\right) \leq \frac{\beta \pi+1}{|z|-1} .
$$

Remark 2.2. Since the matrices $P_{j}$ are nonzero projection matrices, the constant $\beta$ in (2.6) necessarily satisfies $\beta \geq 1$.

We are now ready to formulate the main result of this section.

Theorem 2.3. Let $N \geq 0$ and $A \in \mathbb{C}^{(N+1) \times(N+1)}$ be given by (2.1)-(2.3). The following results hold.

- On the norm of $A^{N}$. If the vectors $\mathbf{u}_{0}, \ldots, \mathbf{u}_{N}$ satisfy

$$
\alpha\left|\sum_{\ell=0}^{N}(-1)^{\ell} \mathbf{u}_{\ell}\right| \leq\left|\sum_{\ell=0}^{N} \mathbf{u}_{\ell}\right|
$$

for some constant $\alpha \geq 0$, then

$$
\left\|A^{N}\right\| \geq \alpha .
$$

- On the resolvent condition. If the vectors $\mathbf{u}_{0}, \ldots, \mathbf{u}_{N}$ satisfy

$$
\left|\sum_{\ell=0}^{j} c_{\ell} \mathbf{u}_{\ell}\right| \leq \beta\left|\sum_{\ell=0}^{N} c_{\ell} \mathbf{u}_{\ell}\right| \quad \text { for all }\left[\begin{array}{c}
c_{0} \\
\vdots \\
c_{N}
\end{array}\right] \in \mathbb{C}^{N+1}, \text { all } j=0, \ldots, N
$$

and some constant $\beta \geq 0$, then

$$
\left\|(z I-A)^{-1}\right\| \leq \frac{\beta \pi+1}{|z|-1} \quad \text { for }|z|>1 .
$$


Proof. We first show that (2.8) holds under the condition (2.7).

Let $\mathbf{p}=\left[(-1)^{0},(-1)^{1}, \ldots,(-1)^{N}\right]^{T} \in \mathbb{C}^{N+1}$ and $\mathbf{q}=[1,1, \ldots, 1]^{T} \in \mathbb{C}^{N+1}$. Note that

$$
\mathbf{q}=\Lambda^{N} \mathbf{p}, \quad T \mathbf{p}=\sum_{\ell=0}^{N}(-1)^{\ell} \mathbf{u}_{\ell} \quad \text { and } \quad T \mathbf{q}=\sum_{\ell=0}^{N} \mathbf{u}_{\ell}
$$

so that $|T \mathbf{q}| \geq \alpha|T \mathbf{p}|$. From $A=T \Lambda T^{-1}$ we obtain $A^{N}=T \Lambda^{N} T^{-1}$, whence

$$
\left\|A^{N}\right\|=\max _{\mathbf{x} \neq 0} \frac{\left|T \Lambda^{N} T^{-1} \mathbf{x}\right|}{|\mathbf{x}|} \geq \frac{\left|T \Lambda^{N} T^{-1}(T \mathbf{p})\right|}{|T \mathbf{p}|}=\frac{|T \mathbf{q}|}{|T \mathbf{p}|} \geq \alpha .
$$

Now assume that (2.9) holds. Given $c_{0}, \ldots, c_{N} \in \mathbb{C}$ let $\mathbf{x}=\sum_{\ell=0}^{N} c_{\ell} \mathbf{u}_{\ell}$ and note that (2.5) implies $c_{\ell}=\mathbf{v}_{\ell}^{T} \mathbf{x}$ for $\ell=0, \ldots, N$. Then

$$
P_{j} \mathbf{x}=\sum_{\ell=0}^{j} \mathbf{u}_{\ell} \mathbf{v}_{\ell}^{T} \mathbf{x}=\sum_{\ell=0}^{j} c_{\ell} \mathbf{u}_{\ell}
$$

so that condition (2.9) is equivalent to the condition $\left|P_{j} \mathbf{x}\right| \leq \beta|\mathbf{x}|$ for all $\mathbf{x} \in \mathbb{C}^{N+1}$ and $j=0, \ldots, N$, i.e., $\left\|P_{j}\right\| \leq \beta$ for all $j=0, \ldots, N$. By Lemma 2.1 this implies (2.10).

Remark 2.4 (on condition (2.7)). If we assume that the coefficients of the vectors $\mathbf{u}_{\ell}$ are nonnegative we expect that cancellations may make the norm of the sum $\sum_{\ell=0}^{N}(-1)^{\ell} \mathbf{u}_{\ell}$ much smaller than of the sum $\sum_{\ell=0}^{N} \mathbf{u}_{\ell}$, thereby leading to a large value of $\alpha$. This justifies the particular choice of the vectors $\mathbf{p}$ and $\mathbf{q}$ in the proof of (2.8).

Remark 2.5. Denoting by $\mathbf{e}_{\ell}$ the $\ell$-th column of the identity matrix $I$, the vectors $\mathbf{p}$ and $\mathbf{q}$ can be written as

$$
\mathbf{p}=\sum_{\ell=0}^{N}(-1)^{\ell} \mathbf{e}_{\ell} \quad \text { and } \quad \mathbf{q}=\sum_{\ell=0}^{N} \mathbf{e}_{\ell}
$$

respectively. In the next section we will introduce functions $p$ and $q$ defined in a similar way in terms of the functions $e_{\ell}(t)=e^{i \ell t}$.

\section{Conditions on a general function $g$}

3.1. Fourier coefficients. We start by recalling some notations and facts related to Fourier theory that will be used in the remainder of the paper.

In all of the following, we mean by a function on the interval $[-\pi, \pi]$, a function which is complex valued and Lebesgue measurable. The class of all Lebesgue integrable functions on $[-\pi, \pi]$ is denoted by $L$.

For integer values $k$ we denote the $k$-th Fourier coefficient of $f \in L$ by

$$
\widehat{f}(k)=\frac{1}{2 \pi} \int_{-\pi}^{\pi} f(t) e^{-i k t} d t .
$$

For $N \geq 0$ we define the $N$-th order Fourier approximation to $f \in L$ by

$$
f_{N}(t)=\sum_{k=-N}^{N} \widehat{f}(k) e^{i k t}
$$

for all $t \in[-\pi, \pi]$. 
We use the notation

$$
\|f\|_{2}=\left\{\frac{1}{2 \pi} \int_{-\pi}^{\pi}|f(t)|^{2} d t\right\}^{1 / 2}
$$

and we denote by $L^{2}$ the class of all functions $f$ on $[-\pi, \pi]$ with $\|f\|_{2}<\infty$. We recall (see, e.g., Zygmund (1979)) that, for any $f \in L^{2}$,

$$
\left\|f_{N}\right\|_{2}=\left\{\sum_{k=-N}^{N}|\widehat{f}(k)|^{2}\right\}^{\frac{1}{2}} \leq\left\{\sum_{k=-\infty}^{\infty}|\widehat{f}(k)|^{2}\right\}^{\frac{1}{2}}=\|f\|_{2} .
$$

Further (see, e.g., Zygmund (1979) again), for $f, g \in L^{2}$, the product $f g$ belongs to $L$ and

$$
\widehat{f g}(\ell)=\sum_{k=-\infty}^{\infty} \widehat{f}(k) \widehat{g}(\ell-k)
$$

The class of all $f \in L$ with $\widehat{f}(k)=0$ whenever $k<0$ will be denoted by $H$. We define

$$
H^{2}=L^{2} \cap H
$$

3.2. The structure of the matrix $T$. We consider the matrix $A$ given by (2.1)2.3, and now assume that $T$ has the lower triangular Toeplitz structure

$$
T=\left[\mathbf{u}_{0}, \mathbf{u}_{1}, \ldots, \mathbf{u}_{N}\right]=\left[\begin{array}{cccc}
d_{0} & & & \\
d_{1} & \ddots & & \\
\vdots & \ddots & \ddots & \\
d_{N} & \ldots & d_{1} & d_{0}
\end{array}\right]
$$

We assume throughout this section that there exists a function $g$ on $[-\pi, \pi]$ such that

$$
g \in H^{2}, \quad 1 / g \in H^{2}, \quad \widehat{g}(\ell)=d_{\ell} \text { for } 0 \leq \ell \leq N \text { with } d_{0} \neq 0 .
$$

The following simple lemma will be used repeatedly.

Lemma 3.1. Assume (3.3)-(3.4). Let $f \in H^{2}$ and $c_{\ell}=\widehat{f}(\ell)$ for $0 \leq \ell \leq N$. Define

$$
\mathbf{x}=\left[\begin{array}{c}
x_{0} \\
\vdots \\
x_{N}
\end{array}\right]=\sum_{\ell=0}^{N} c_{\ell} \mathbf{u}_{\ell} .
$$

Then $x_{\ell}$ is equal to the $\ell$-th Fourier coefficient of $f g$, i.e.,

$$
x_{\ell}=\widehat{f g}(\ell) \quad \text { for } \ell=0, \ldots, N \text {. }
$$

Proof. From (3.3) we have $x_{\ell}=\sum_{k=0}^{\ell} c_{k} d_{\ell-k}$. In view of (3.2) and (3.4) we conclude that $x_{\ell}$ is equal to the $\ell$-th Fourier coefficient of $f g$. 
3.3. Transforming (2.7). We define the functions $p$ and $q$ by

$$
p(t)=\sum_{\ell=0}^{N}(-1)^{\ell} e^{i \ell t}=\frac{1+(-1)^{N} e^{i(N+1) t}}{1+e^{i t}}
$$

and

$$
q(t)=\sum_{\ell=0}^{N} e^{i \ell t}=\frac{1-e^{i(N+1) t}}{1-e^{i t}}
$$

respectively, which are analogous to the vectors $\mathbf{p}$ and $\mathbf{q}$ introduced in Section 2 (see Remark 2.5). The following lemma transforms (2.7) into a condition which involves functions on $[-\pi, \pi]$ and their $L^{2}$ norm, rather than vectors in $\mathbb{C}^{N+1}$ and their Euclidean norm.

Lemma 3.2. Assume (3.3)-(3.6). Then (2.7) is equivalent to

$$
\alpha\left\|(p g)_{N}\right\|_{2} \leq\left\|(q g)_{N}\right\|_{2} \text {. }
$$

Proof. Let

$$
\mathbf{x}=\left[\begin{array}{c}
x_{1} \\
\vdots \\
x_{N}
\end{array}\right]=\sum_{\ell=0}^{N}(-1)^{\ell} \mathbf{u}_{\ell} \quad \text { and } \quad \mathbf{y}=\left[\begin{array}{c}
y_{1} \\
\vdots \\
y_{N}
\end{array}\right]=\sum_{\ell=0}^{N} \mathbf{u}_{\ell} .
$$

Denoting the Fourier coefficients of $p g$ and $q g$ by $a_{\ell}$ and $b_{\ell}$, respectively, we have, by Lemma 3.1 .

$$
x_{\ell}=a_{\ell} \quad \text { and } \quad y_{\ell}=b_{\ell} .
$$

Since

$$
|\mathbf{x}|=\left(\sum_{\ell=0}^{N}\left|x_{\ell}\right|^{2}\right)^{\frac{1}{2}}=\left(\sum_{\ell=0}^{N}\left|a_{\ell}\right|^{2}\right)^{\frac{1}{2}}=\left\|(p g)_{N}\right\|_{2}
$$

and

$$
|\mathbf{y}|=\left(\sum_{\ell=0}^{N}\left|y_{\ell}\right|^{2}\right)^{\frac{1}{2}}=\left(\sum_{\ell=0}^{N}\left|b_{\ell}\right|^{2}\right)^{\frac{1}{2}}=\left\|(q g)_{N}\right\|_{2}
$$

we conclude that (2.7) is equivalent to the inequality (3.7).

3.4. Transforming (2.9). In order to obtain a sufficient condition for (2.9) we assume that $0 \leq j \leq N$ and that $c_{0}, \ldots, c_{N} \in \mathbb{C}$ are given. Let

$$
\mathbf{x}=\left[\begin{array}{c}
x_{1} \\
\vdots \\
x_{N}
\end{array}\right]=\sum_{\ell=0}^{j} c_{\ell} \mathbf{u}_{\ell} \quad \text { and } \quad \mathbf{y}=\left[\begin{array}{c}
y_{1} \\
\vdots \\
y_{N}
\end{array}\right]=\sum_{\ell=0}^{N} c_{\ell} \mathbf{u}_{\ell} .
$$

In view of (3.4) we define a function $f$ on $[-\pi, \pi]$ by

$$
f(t)=\frac{\sum_{\ell=0}^{N} y_{\ell} e^{i \ell t}}{g(t)}
$$

so that

$$
|\mathbf{y}|=\|f g\|_{2}, \quad f \in H^{2} .
$$


From Lemma 3.1 we conclude that the $N+1$ components $z_{\ell}$ of the vector

$$
\mathbf{z}=\sum_{\ell=0}^{N} \widehat{f}(\ell) \mathbf{u}_{\ell}
$$

are equal to the corresponding Fourier coefficients of $f g$. Since these coefficients are equal to $y_{\ell}(0 \leq \ell \leq N)$, we have $\mathbf{z}=\mathbf{y}$. Consequently,

$$
c_{\ell}=\widehat{f}(\ell) \text { for } 0 \leq \ell \leq N .
$$

Applying Lemma 3.1 once more, in combination with the last equality, we see that $x_{\ell}$ equals the $\ell$-th Fourier coefficient of $f_{j} g$, so that

$$
|\mathbf{x}|=\left\|\left(f_{j} g\right)_{N}\right\|_{2} .
$$

From (3.8)-3.10) we obtain the following result.

Lemma 3.3. Assume (3.3)-(3.4). Suppose $\beta$ is a constant such that

$$
\left\|\left(f_{j} g\right)_{N}\right\|_{2} \leq \beta\|f g\|_{2} \quad \text { for all } f \in H^{2} \text { and } j=0,1, \ldots, N \text {. }
$$

Then (2.9) holds.

3.5. Conditions on $g$. Combining Theorem 2.3 and Lemmas 3.2 and 3.3 we obtain, in view of (3.1), the following main conclusion of Section 3 .

Theorem 3.4. Let $N \geq 0$ and $A \in \mathbb{C}^{(N+1) \times(N+1)}$ be given by (2.1) - (2.2.2), (3.3)(3.4). The following results hold.

- On the norm of $A^{N}$ : If the functions $p$ and $q$ given by (3.5)-(3.6) satisfy

$$
\alpha\|p g\|_{2} \leq\left\|(q g)_{N}\right\|_{2}
$$

for some constant $\alpha \geq 0$, then (2.8) holds.

- On the resolvent condition: If

$$
\left\|f_{j} g\right\|_{2} \leq \beta\|f g\|_{2} \quad \text { for all } f \in H^{2} \text { and } j=0,1,2, \ldots
$$

and some constant $\beta \geq 0$, then (2.10) holds.

\section{Conditions on a SPECial Function $g$}

4.1. Choice of a function $g$ satisfying (3.11)-(3.12). When applying Theorem 3.4 in proving a "large" lower bound for $\left\|A^{N}\right\|$, it is necessary to choose $g$ such that a large value for $\alpha$ in (3.11) is possible. Therefore, it is natural to choose $g$ such that the ratio $\frac{\|q g\|_{2}}{\|p g\|_{2}}$ is large. Noting that

$$
|p(\pi)|=\max _{t}|p(t)| \quad \text { and } \quad|q(0)|=\max _{t}|q(t)|,
$$

one is led to choosing $g$ such that $|g(t)|$ is large for $t \simeq 0$ and small for $t \simeq \pi$. We make the following choice:

$$
g(t)=\left(\frac{1+e^{i t}}{1-e^{i t}}\right)^{\gamma} \quad \text { for } 0<|t|<\pi, \quad \text { with } 0<\gamma<\frac{1}{2}
$$

(see Figure 1). In the following, $\gamma$ will depend on a given parameter $\epsilon>0$, i.e., $\gamma=\gamma(\epsilon)$. The exact dependence of $\gamma$ on $\epsilon$ will be given explicitly below. 


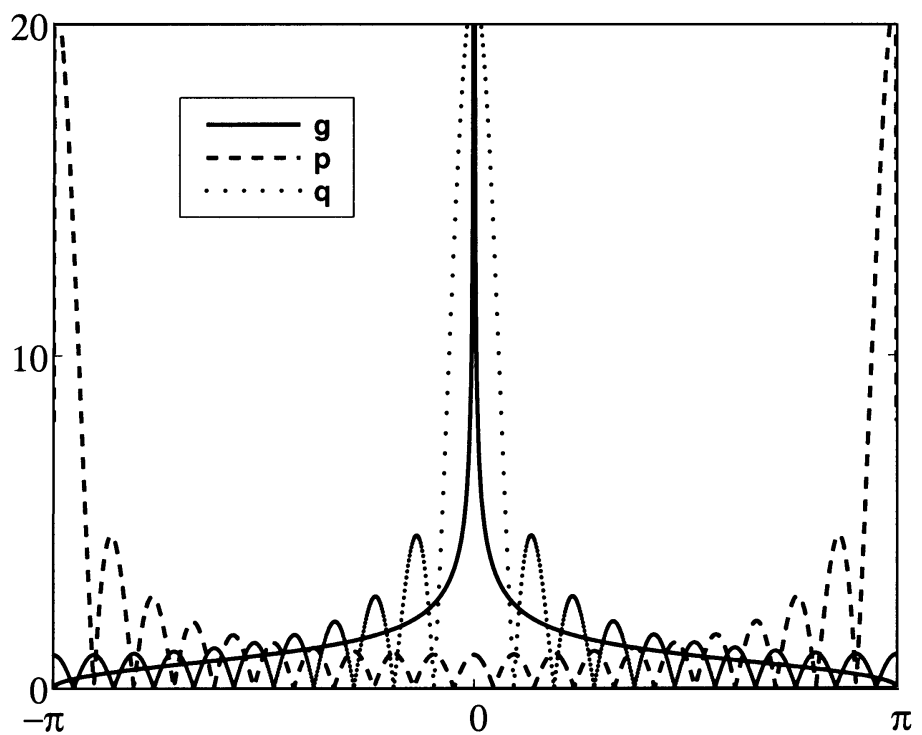

Figure 1. Plot of the magnitude of the functions $g, p$ and $q$ given by (4.1), 3.5 and (3.6) with $\gamma=0.49$.

Clearly, both $g$ and $1 / \mathrm{g}$ belong to the space $L^{2}$. Further let $\mathcal{C}$ be any simple positively oriented smooth curve contained in the closed unit disk, with $z=0$ in its interior and $z=1$ in its exterior. Then, for all integer values $k$, we have

$$
\widehat{g}(k)=\frac{1}{2 \pi} \int_{-\pi}^{\pi}\left(\frac{1+e^{i t}}{1-e^{i t}}\right)^{\gamma} e^{-i k t} d t=\frac{1}{2 \pi i} \oint_{\mathcal{C}}\left(\frac{1+z}{1-z}\right)^{\gamma} \frac{d z}{z^{k+1}} .
$$

From this we see that $\widehat{g}(k)=0$ for $k<0$ and $d_{0}=\widehat{g}(0)=1$. Hence $g \in H^{2}$. Since $(g(t))^{-1}=g(t+\pi)$ for $0<|t|<\pi$, we also have $1 / g \in H^{2}$. Thus $g$ satisfies 3.4.

In this section we shall show that, for a proper choice of $\gamma(\epsilon)$, the function $g$ also satisfies the conditions (3.11) and 3.12 with $\alpha=\mathcal{O}\left(N^{1-\epsilon}\right)$ and $\beta=\mathcal{O}(1)$, respectively.

4.2. The function $g$ satisfies (3.12). A set of sufficient conditions for a general function $g \in H^{2}$ to satisfy (3.12) was recently given in Borovykh \& Spijker (2001). These conditions are satisfied in particular when the function $g \in H^{2}$ has a polar decomposition given by

$$
g(t)=|g(t)| e^{i \theta(t)} \quad \text { with } \quad 2|\theta(t)| \leq \sigma \text { for almost all } t \in[-\pi, \pi]
$$

with some constant $\sigma<\frac{\pi}{2}$. For such functions $g$ it was shown that (3.12) holds with $\beta=(\cos \sigma)^{-1}$. We refer the reader to Borovykh \& Spijker (2001) for a precise formulation of the more general statement. 
The function $g$ defined by (4.1) can be written in polar form as

$$
g(t)=\left(i \cot \frac{t}{2}\right)^{\gamma}=|g(t)| e^{i \theta(t)}
$$

with $\theta(t)=\gamma \frac{\pi}{2} \operatorname{sign}(t)$. Thus

$$
2|\theta(t)| \leq \gamma \pi<\frac{\pi}{2}
$$

for any $t \in(-\pi, \pi) \backslash\{0\}$. Consequently, (4.2) holds with $\sigma=\gamma \pi$ and (3.12) holds with

$$
\beta=(\cos \gamma \pi)^{-1}
$$

4.3. The function $g$ satisfies (3.11). We now show that, for $0<\epsilon<1$, the condition (3.11) holds with $\alpha=\mathcal{O}\left(N^{1-\epsilon}\right)$ provided the function $\gamma=\gamma(\epsilon)$ is chosen appropriately. We proceed by first determining an upper bound for $\|p g\|_{2}$ and then a lower bound for $\left\|(q g)_{N}\right\|_{2}$.

Lemma 4.1. We have

$$
\|p g\|_{2} \leq \frac{2}{\sqrt{1-2 \gamma}}(N+1)^{\frac{1}{2}-\gamma} .
$$

Proof. Using the $2 \pi$-periodicity of the integrand,

$$
\begin{aligned}
\|p g\|_{2}^{2} & =\frac{1}{2 \pi} \int_{-\pi}^{\pi}|p(t) g(t)|^{2} d t \\
& =\frac{1}{2 \pi} \int_{0}^{2 \pi}|p(t) g(t)|^{2} d t \\
& =\frac{1}{2 \pi} \int_{-\pi}^{\pi}|p(t+\pi) g(t+\pi)|^{2} d t \\
& =\frac{1}{\pi} \int_{0}^{\pi} \frac{\left(\sin \frac{(N+1) t}{2}\right)^{2}}{\left(\cos \frac{t}{2}\right)^{2 \gamma}\left(\sin \frac{t}{2}\right)^{2-2 \gamma}} d t \\
& =I_{1}+I_{2}
\end{aligned}
$$

with

$$
I_{1}=\frac{1}{\pi} \int_{0}^{\frac{\pi}{2}} \frac{\left(\sin \frac{(N+1) t}{2}\right)^{2}}{\left(\cos \frac{t}{2}\right)^{2 \gamma}\left(\sin \frac{t}{2}\right)^{2-2 \gamma}} d t
$$

and

$$
I_{2}=\frac{1}{\pi} \int_{\frac{\pi}{2}}^{\pi} \frac{\left(\sin \frac{(N+1) t}{2}\right)^{2}}{\left(\cos \frac{t}{2}\right)^{2 \gamma}\left(\sin \frac{t}{2}\right)^{2-2 \gamma}} d t .
$$

On one hand we obtain, using the fact that

$$
\frac{\sqrt{2}}{\pi} t \leq \sin \frac{t}{2} \quad \text { for } 0 \leq t \leq \frac{\pi}{2}
$$


and $0<\gamma<\frac{1}{2}$

$$
\begin{aligned}
I_{1} & \leq \frac{1}{\pi} \int_{0}^{\frac{\pi}{2}} \frac{\left(\sin \frac{(N+1) t}{2}\right)^{2}}{\left(\cos \frac{\pi}{4}\right)^{2 \gamma}\left(\frac{\sqrt{2}}{\pi} t\right)^{2-2 \gamma}} d t \\
& =\left(\frac{\pi}{2}\right)^{1-2 \gamma} \int_{0}^{\frac{\pi}{2}} t^{2 \gamma-2}\left(\sin \frac{(N+1) t}{2}\right)^{2} d t \\
& =\left(\frac{\pi(N+1)}{4}\right)^{1-2 \gamma} \int_{0}^{\frac{(N+1) \pi}{4}} s^{2 \gamma-2}(\sin s)^{2} d s \\
& \leq(N+1)^{1-2 \gamma}\left(\int_{0}^{1} s^{2 \gamma} d s+\int_{1}^{\infty} s^{2 \gamma-2} d s\right) \\
& =\frac{2}{1-4 \gamma^{2}}(N+1)^{1-2 \gamma} .
\end{aligned}
$$

On the other hand the integral $I_{2}$ can be bounded, using (4.5) again, by

$$
\begin{aligned}
I_{2} & \leq \frac{1}{\pi} \int_{\frac{\pi}{2}}^{\pi} \frac{d t}{\left(\cos \frac{t}{2}\right)^{2 \gamma}\left(\sin \frac{\pi}{4}\right)^{2-2 \gamma}} \\
& =\frac{2^{1-\gamma}}{\pi} \int_{0}^{\frac{\pi}{2}} \frac{d s}{\left(\sin \frac{s}{2}\right)^{2 \gamma}} \\
& \leq \frac{2^{1-\gamma}}{\pi} \int_{0}^{\frac{\pi}{2}} \frac{d s}{\left(\frac{\sqrt{2}}{\pi} s\right)^{2 \gamma}} \\
& =\frac{1}{1-2 \gamma} .
\end{aligned}
$$

Combining the above upper bounds we get $\|p g\|_{2}^{2}=I_{1}+I_{2} \leq \frac{3}{1-2 \gamma}(N+1)^{1-2 \gamma}$ which yields (4.4).

The following lemma gives a lower bound for $\left\|(g p)_{N}\right\|_{2}$.

Lemma 4.2. We have

$$
\left\|(q g)_{N}\right\|_{2} \geq \frac{(N+1)^{\frac{1}{2}+\gamma}}{2}-\frac{1}{\sqrt{1-2 \gamma}} .
$$

Proof. We write

$$
g(t)=g_{1}(t)\left(1+g_{2}(t)\right)
$$

with

$$
g_{1}(t)=\left(\frac{2}{1-e^{i t}}\right)^{\gamma} \quad \text { and } \quad g_{2}(t)=\left(\frac{1+e^{i t}}{2}\right)^{\gamma}-1 .
$$

The goal of the decomposition (4.7) is to replace the evaluation of the Fourier coefficients $\widehat{q g}(k)$ of $q g$ by those of the function $q g_{1}$, which are simpler to derive and bound. A lower bound for $\left\|\left(q g_{1}\right)_{N}\right\|_{2}$ and an upper bound for $\left\|\left(q g_{1} g_{2}\right)_{N}\right\|_{2}$ which are suitable can then be used to bound $\left\|(q g)_{N}\right\|_{2}$ using the triangular inequality

$$
\left\|(q g)_{N}\right\|_{2}=\left\|\left(q g_{1}\right)_{N}+\left(q g_{1} g_{2}\right)_{N}\right\|_{2} \geq\left\|\left(q g_{1}\right)_{N}\right\|_{2}-\left\|\left(q g_{1} g_{2}\right)_{N}\right\|_{2} .
$$


A lower bound for $\left\|\left(q g_{1}\right)_{N}\right\|_{2}$ is first derived by considering the Fourier coefficients of $q g_{1}$. With the same curve $\mathcal{C}$ used in subection 4.1, we have, for $k \geq 0$,

$$
\begin{aligned}
\widehat{q g}_{1}(k) & =\frac{1}{2 \pi} \int_{-\pi}^{\pi} g_{1}(t) e^{-i k t} d t \\
& =\frac{1}{2 \pi i} \oint_{\mathcal{C}}\left(\frac{2}{1-z}\right)^{\gamma} \frac{1-z^{N+1}}{1-z} z^{-(k+1)} d z \\
& =2^{\gamma} \frac{\left[\frac{1-z^{N+1}}{(1-z)^{1+\gamma}}\right]^{(k)}(0)}{k !} .
\end{aligned}
$$

We thus obtain $\widehat{q g}_{1}(0)=2^{\gamma}$ and for $1 \leq k \leq N$,

$$
\widehat{q g}_{1}(k)=2^{\gamma} \frac{\left[(1-z)^{-(1+\gamma)}\right]^{(k)}(0)}{k !}=2^{\gamma} \frac{(1+\gamma)(2+\gamma) \cdots(k+\gamma)}{k !}=2^{\gamma} \prod_{\ell=1}^{k}\left(1+\frac{\gamma}{\ell}\right) .
$$

Note that $\widehat{q g_{1}}(k)>0$ for all $k \geq 0$. Moreover,

$$
\begin{aligned}
\log \prod_{\ell=1}^{k}\left(1+\frac{\gamma}{\ell}\right) & =\sum_{\ell=1}^{k} \log \left(1+\frac{\gamma}{\ell}\right) \\
& \geq \sum_{\ell=1}^{k}\left(\frac{\gamma}{\ell}-\frac{\gamma^{2}}{2 \ell^{2}}\right) \\
& \geq \gamma \log (k+1)-\frac{\gamma^{2} \pi^{2}}{12}
\end{aligned}
$$

so that, for $k \geq 0$,

$$
\widehat{q g_{1}}(k) \geq 2^{\gamma} e^{-\frac{\gamma^{2} \pi^{2}}{12}}(k+1)^{\gamma}
$$

We are now in position to evaluate $\left\|\left(q g_{1}\right)_{N}\right\|_{2}$. From (3.1) we obtain

$$
\begin{aligned}
\left\|\left(q g_{1}\right)_{N}\right\|_{2}^{2} & =\sum_{k=0}^{N}\left|\widehat{q g_{1}}(k)\right|^{2} \\
& \geq 4^{\gamma} e^{-\frac{\gamma^{2} \pi^{2}}{6}} \sum_{k=0}^{N}(k+1)^{2 \gamma} \\
& \geq 4^{\gamma} e^{-\frac{\gamma^{2} \pi^{2}}{6}} \int_{0}^{N+1} t^{2 \gamma} d t \\
& =\frac{4^{\gamma} e^{-\frac{\gamma^{2} \pi^{2}}{6}}}{1+2 \gamma}(N+1)^{1+2 \gamma} \\
& \geq \frac{(N+1)^{1+2 \gamma}}{4}
\end{aligned}
$$

(since $0<\gamma<\frac{1}{2}$ ). Thus

$$
\left\|\left(q g_{1}\right)_{N}\right\|_{2} \geq \frac{(N+1)^{\frac{1}{2}+\gamma}}{2} .
$$

To obtain an upper bound for $\left\|\left(q g_{1} g_{2}\right)_{N}\right\|_{2}$ we first note that

$$
g_{2}(t)=\left[\left(s \frac{1+e^{i t}}{2}+1-s\right)^{\gamma}\right]_{s=0}^{s=1}=\gamma \frac{e^{i t}-1}{2} \int_{0}^{1}\left(s \frac{1+e^{i t}}{2}+1-s\right)^{\gamma-1} d s
$$


so that (since $\gamma-1<0$ )

$$
\left|g_{2}(t)\right| \leq \gamma\left|\frac{e^{i t}-1}{2}\right| \int_{0}^{1}\left|\frac{s}{2}-\left(1-\frac{s}{2}\right)\right|^{\gamma-1} d s=\left|\frac{e^{i t}-1}{2}\right| .
$$

Using (3.1) and (4.10) we obtain

$$
\begin{aligned}
\left\|\left(q g_{1} g_{2}\right)_{N}\right\|_{2}^{2} & \leq\left\|q g_{1} g_{2}\right\|_{2}^{2} \\
& =\frac{1}{2 \pi} \int_{-\pi}^{\pi}\left|q(t) g_{1}(t) g_{2}(t)\right|^{2} d t \\
& \leq \frac{1}{8 \pi} \int_{-\pi}^{\pi} \frac{\left|1-e^{i(N+1) t}\right|^{2}}{\left|\frac{1-e^{i t}}{2}\right|^{2 \gamma}} d t \\
& \leq \frac{1}{\pi} \int_{0}^{\pi} \frac{d t}{\left|\sin \frac{t}{2}\right|^{2 \gamma}} \\
& \leq \pi^{2 \gamma-1} \int_{0}^{\pi} t^{-2 \gamma} d t \\
& =\frac{1}{1-2 \gamma} .
\end{aligned}
$$

Thus

$$
\left\|\left(q g_{1} g_{2}\right)_{N}\right\|_{2} \leq \frac{1}{\sqrt{1-2 \gamma}} .
$$

Combining (4.8), (4.9) and (4.11) yields the result.

For $0<\epsilon<1$ given we now choose

$$
\gamma=\frac{1-\epsilon}{2}
$$

By Lemmas 4.1 and 4.2 we then obtain the bound

$$
\frac{\left\|(q g)_{N}\right\|_{2}}{\|p g\|_{2}} \geq \frac{\frac{1}{2}(N+1)^{\frac{1}{2}+\gamma}-\frac{1}{\sqrt{1-2 \gamma}}}{\frac{2}{\sqrt{1-2 \gamma}}(N+1)^{\frac{1}{2}-\gamma}} \geq \frac{\sqrt{\epsilon}}{4}(N+1)^{1-\epsilon}-\frac{1}{2}
$$

4.4. Main conclusion. We now state the main conclusion of Section 4

Theorem 4.3. Let $N \geq 0,0<\epsilon<1$ and $A \in \mathbb{C}^{(N+1) \times(N+1)}$ be given by

$$
A=\left[\begin{array}{cccc}
d_{0} & & & \\
d_{1} & \ddots & & \\
\vdots & \ddots & \ddots & \\
d_{N} & \ldots & d_{1} & d_{0}
\end{array}\right]\left[\begin{array}{llll}
1 & & & \\
& e^{\frac{i \pi}{N}} & & \\
& & \ddots & \\
& & e^{\frac{i N \pi}{N}}
\end{array}\right]\left[\begin{array}{cccc}
d_{0} & & & \\
d_{1} & \ddots & & \\
\vdots & \ddots & \ddots & \\
& & & \\
d_{N} & \ldots & d_{1} & d_{0}
\end{array}\right]^{-1},
$$

where $d_{\ell}$ is the $\ell$-th Fourier coefficient of the function

$$
g(t)=\left(\frac{1+e^{i t}}{1-e^{i t}}\right)^{\frac{1-\epsilon}{2}} .
$$

Then A satisfies the following inequalities. 
- On the norm of $A^{N}$ :

$$
\left\|A^{N}\right\| \geq \frac{\sqrt{\epsilon}}{6}(N+1)^{1-\epsilon} .
$$

- On the resolvent condition:

$$
\left\|(z I-A)^{-1}\right\| \leq \frac{\frac{\pi}{\sin \frac{\epsilon \pi}{2}}+1}{|z|-1} \quad \text { for }|z|>1 .
$$

Proof. Combining Theorem 3.4 and the relations (4.3), (4.12) and (4.13), we obtain (4.15) and $\left\|A^{N}\right\| \geq \frac{\sqrt{\epsilon}}{4}(N+1)^{1-\epsilon}-\frac{1}{2}$. Since the right-hand side of the latter inequality is larger than or equal to $\frac{\sqrt{\epsilon}}{4}(N+1)^{1-\epsilon}-\frac{1}{2}\left\|A^{N}\right\|$, we obtain (4.14).

We finally note that Theorem 1.2 follows directly from Theorem 4.3 .

4.5. About the coefficients $d_{\ell}$ occurring in Theorem 4.3. The coefficients $d_{\ell}$ of the Toeplitz matrix $T$ of Theorem 4.3 can be determined explicitly from

$$
\begin{aligned}
\left(\frac{1+z}{1-z}\right)^{\gamma} & =\left[\sum_{\ell \geq 0}\left(\begin{array}{l}
\gamma \\
\ell
\end{array}\right) z^{\ell}\right] \cdot\left[\sum_{k \geq 0}\left(\begin{array}{c}
\gamma+k-1 \\
k
\end{array}\right) z^{k}\right] \\
& =\sum_{\ell \geq 0}\left[\sum_{k=0}^{\ell}\left(\begin{array}{c}
\gamma+k-1 \\
k
\end{array}\right)\left(\begin{array}{c}
\gamma \\
\ell-k
\end{array}\right)\right] z^{\ell},
\end{aligned}
$$

where $\gamma=\frac{1-\epsilon}{2}$. Then we have

$$
d_{\ell}=\sum_{k=0}^{\ell}\left(\begin{array}{c}
\gamma+k-1 \\
k
\end{array}\right)\left(\begin{array}{c}
\gamma \\
\ell-k
\end{array}\right) .
$$

The values of $d_{\ell}$ for $0 \leq \ell \leq 100$ are shown in Figure 2 for $\epsilon=0.02$, i.e., $\gamma=0.49$. Note that all $d_{\ell}$ 's are positive and slowly decreasing down to 0 as $\ell$ increases.

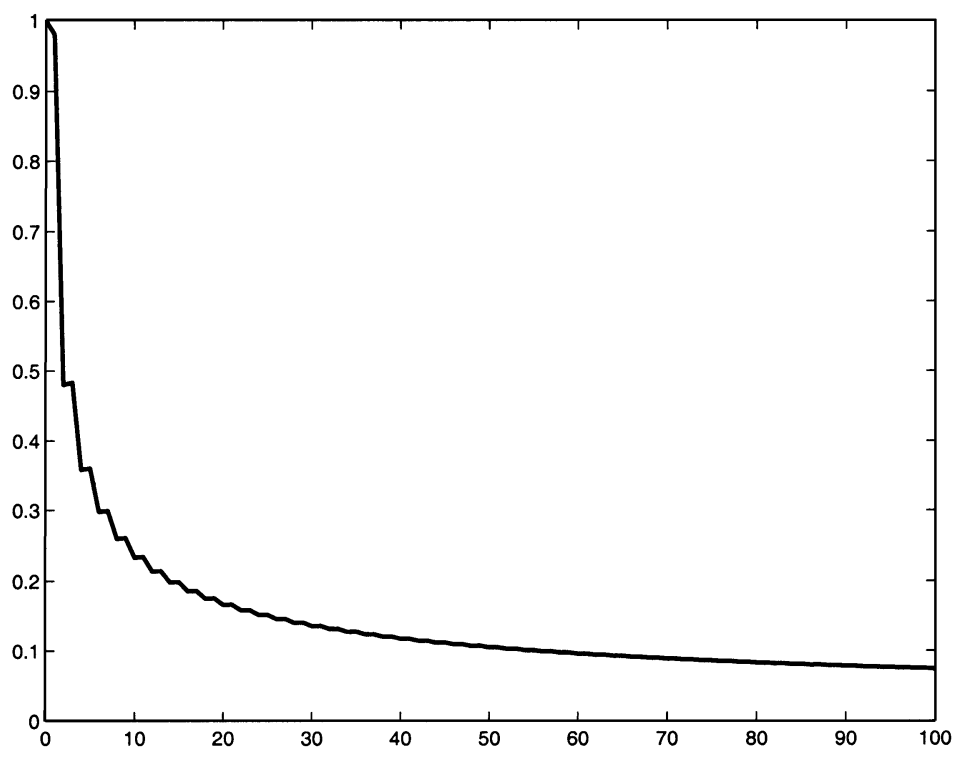

Figure 2. Coefficients $d_{\ell}$ vs $\ell$ for $\epsilon=0.02$. 


\section{ACKNOWLEDGMENTS}

The first author is most thankful to L.N. Trefethen for a stimulating discussion in 1991 about the problem treated in this paper.

This work started while the last two authors were visiting the University of Leiden, The Netherlands, in 1997 and was completed during the first author's visit to Arizona State University, USA, in the Spring of 1998.

\section{REFERENCES}

1. Borovykh N., Spijker M.N. (2000): Resolvent conditions and bounds on the powers of matrices, with relevance to numerical stability of initial value problems, J. Comput. Appl. Math., 125, 41-56. MR 2001k:65099

2. Borovykh N., Spijker M.N. (2001): Bounding partial sums of Fourier series in weighted $L^{2}$ norms, with applications to matrix analysis, to appear in J. Comput. Appl. Math.

3. Dorsselaer J.L.M. van, Kraaijevanger J.F.B.M., Spijker M.N. (1993): Linear stability analysis in the numerical solution of initial value problems, Acta Numerica 1993, 199-237. MR 94e:65051

4. Giles M.B. (1997): On the stability and convergence of discretizations of initial value p.d.e.'s, IMA Jour. Numer. Anal., 17, 563-576. MR 98j:65061

5. Kraaijevanger J.F.B.M. (1994): Two counterexamples related to the Kreiss matrix theorem, BIT 34, 113-119. MR 98c:65154

6. Kreiss H.-O. (1962): Über die Stabilitätsdefinition für Differenzengleichungen die partielle Differentialgleichungen approximieren, BIT 2, 153-181. MR 99:2992

7. LeVeque R.J., Trefethen L.N. (1984): On the resolvent condition in the Kreiss matrix theorem, BIT 24, 584-591. MR 86c:39004

8. Lubich Ch., Nevanlinna O. (1991): On resolvent conditions and stability estimates, BIT 31, 293-313. MR 92h:65145

9. McCarthy C.A., Schwartz J. (1965): On the norm of a finite boolean algebra of projections, and applications to theorems of Kreiss and Morton, Comm. Pure Appl. Math. 18, 191-201. MR 31:5097

10. Nevanlinna O. (1997): On the growth of the resolvent operators for power bounded operators, in Linear Operators, Banach Center Publications, Volume 38, Inst. Math. Pol. Acad. Sciences (Warsaw), 247-264. MR 98e:47006

11. Reddy S.C., Trefethen L.N. (1990): Lax-stability of fully discrete spectral methods via stability regions and pseudo-eigenvalues, Comp. Meth. Appl. Mech. Eng. 80, 147-164. MR 91j:65102

12. Reddy S.C., Trefethen L.N. (1992): Stability of the method of lines, Numer. Math. 62, 235267. MR 93d:65086

13. Sand J. (1996): On some stability bounds subject to Hille-Yosida resolvent conditions, BIT 36, 378-386. MR 99c:65169

14. Shields A.L. (1978): On Möbius bounded operators, Acta Sci. Math. 40, 371-374. MR 80a: 47029

15. Spijker M.N. (1991): On a conjecture by LeVeque and Trefethen related to the Kreiss matrix theorem, BIT 31, 551-555. MR 92h:15012

16. Spijker M.N., Straetemans F.A.J. (1996): Stability estimates for families of matrices of nonuniformly bounded order, Linear Algebra Appl. 239, 77-102. MR 98g:65041

17. Spijker M.N., Straetemans F.A.J. (1997): Error growth analysis, via stability regions, for discretizations of initial value problems, BIT 37, 442-464. MR 98g:65066

18. Strikwerda J.C., Wade B.A. (1991): Cesaro means and the Kreiss matrix theorem, Linear Algebra Appl. 145, 89-106. MR 91m:15028

19. Strikwerda J.C., Wade B.A. (1997): A survey of the Kreiss matrix theorem for power bounded families of matrices and its extensions, in Linear Operators, Banach Center Publications, Volume 38, Inst. Math. Pol. Acad. Sciences (Warzaw), 329-360. MR 98f:15020

20. Toh K-C, Trefethen L.N. (1999): The Kreiss matrix theorem on a general complex domain, SIAM J. Matrix Anal. Appl. 21, 145-165. MR 2000h:65054

21. Wegert E., Trefethen L.N. (1994): From the Buffon needle problem to the Kreiss matrix theorem, Amer. Math. Monthly 101, 132-139. MR 95b:30036 
22. Zygmund A. (1979): Trigonometric Series, Vol. I, Cambridge University Press (Cambridge). MR 89c:42001

Department of Mathematics, Rijksuniversiteit Leiden, P.O. Box 9512, NL 2300 RA Leiden, The Netherlands

E-mail address: spijker@math.leidenuniv.nl

$U R L$ : www.math.leidenuniv.nl/ spijker

Department of Mathematics, Arizona State University, Tempe, Arizona 85287-1804

E-mail address: tracogna@math.la.asu.edu

$U R L$ : www.math.asu.edu/ tracogna

Department of Mathematics, Arizona State University, Tempe, Arizona 85287-1804

E-mail address: bdw@math.asu.edu

$U R L$ : math.la.asu.edu/ ${ }^{b d w}$ 